\title{
Quantized separations of phase-locked soliton pairs in fiber lasers
}

\author{
J. M. Soto-Crespo \\ Instituto de Óptica, Consejo Superior de Investigaciones Cientificas, Serrano 121, 28006 Madrid, Spain
}

Nail Akhmediev

Optical Sciences Centre, Research School of Physical Sciences and Engineering, The Australian National University, Canberra ACT 0200, Australia

Ph. Grelu and F. Belhache

Laboratoire de Physique de l'Université de Bourgogne, Unité Mixte de Recherche 5027, B.P. 47870, 21078 Dijon, France

Received January 8, 2003

We report the discovery of a quantization of the separation between phase-locked soliton pairs that is related to the radiation waves known as Kelly sidebands, in a passively mode-locked fiber ring laser. Our numerical simulations that predict this phenomenon have been confirmed by our experimental results. @ 2003 Optical Society of America

OCIS codes: $\quad 060.5530,140.3510$.

Multiple-pulse generation is a well-known phenomenon in passively mode-locked laser systems that appears when the gain exceeds certain thresholds. Formation of double, triple, and more pulses has been experimentally observed. ${ }^{1-5}$ In some cases these pulses interact through their tails and form a stable solution with a fixed distance and a fixed phase difference. ${ }^{2}$ The formation of these pairs with a $\pi / 2$ phase difference had been predicted theoretically in the framework of the quintic complex Ginzburg-Landau equation. ${ }^{6}$

There are various mechanisms responsible for coupling the adjacent pulses together. For example, longrange soliton interaction through acoustic waves in a fiber has been studied in a number of publications. ${ }^{7,8}$ Stabilized pulse spacing as a result of gain depletion and recovery was reported in Ref. 9. Long-range soliton interactions in periodically amplified fiber links was considered in Ref. 10. Interaction through the radiation field occurs even in the integrable models. ${ }^{11}$

In this Letter we study in detail the process of double-pulse generation in the model of a passively mode-locked system with parameter management. The main feature of the system is the periodicity of the medium parameters for the pulse propagating inside the cavity. As a result, the pulse changes along with propagation in the cavity, but at any fixed point the pulse shape converges in time to a stationary profile. Another important property of the model is that it is creating Kelly sidebands in the spectrum as a result of periodic pulse reshaping. These sidebands are small-amplitude radiative waves emitted by the pulse because of the periodicity of the processes in the cavity. This radiation in turn can influence the interaction between the solitons and relates their phases and separations. We have found in our simulations that solitons can see each other at relatively larger distances than they would in the absence of radiation. This explanation is further corroborated by our experiments.

The choice of the system parameters is dictated by the experimental setup described in Ref. 2 . The model is shown in Fig. 1. It consists of an isotropic erbium-doped fiber (EDF), a quarter-wavelength plate, a birefringent single-mode fiber (SMF), and a polarizer. Linear losses are incorporated between these elements at points $a-d$. The wave propagation in the EDF is described by the nonlinear Schrödinger equation with an additional gain term:

$$
i U_{z}+\frac{D}{2} U_{t t}+\Gamma|U|^{2} U=i g(Q) U+i \beta U_{t t},
$$

where $D=\beta_{2}^{\mathrm{Er}} / \beta_{2}^{\mathrm{SMF}}$ is the dimensionless dispersion parameter. $\beta_{2}^{\mathrm{Er}}$ and $\beta_{2}^{\mathrm{SMF}}$ are the dispersion coefficients related to the two pieces of fiber, respectively. $\Gamma=A_{\text {eff }}^{\mathrm{SMF}} / A_{\text {eff }}^{\mathrm{Er}}\left(A_{\text {eff }}\right.$ is the effective area in each type of fiber), and $\beta$ is the strength of the spectral filtering as a result of the gain-limited bandwidth. The function $g(Q)$ represents gain in the EDF, $g(Q)=g_{0}(z) /(1+$ $\left.Q / E_{L}\right)$, where $g_{0}(z)=g_{0 i}+\left(g_{0 f}-g_{0 i}\right) z / L_{\mathrm{Er}}$, and $Q=$ $\int_{-\infty}^{\infty}|U|^{2} \mathrm{~d} t, L_{\mathrm{Er}}$ is the length of the EDF, $E_{L}$ is the saturation energy, and $g_{0}$ is the small-signal gain, which depends on $z$ because of the pump depletion.

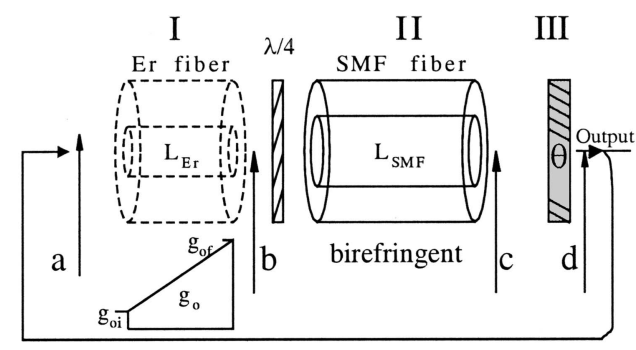

Fig. 1. Model of a passively mode-locked fiber laser used in the simulations. 
For simplicity we adopt linear polarization all along the EDF. Nonlinear birefringence, which is required for pulse formation in this laser, is acting during propagation in the passive SMF fiber, as described by the two following coupled nonlinear Schrödinger equations:

$$
\begin{aligned}
& i \phi_{z}+\gamma \phi+\frac{1}{2} \phi_{t t}+|\phi|^{2} \phi+\frac{2}{3}|\psi|^{2} \phi+\frac{1}{3} \psi^{2} \phi^{*}=0, \\
& i \psi_{z}-\gamma \psi+\frac{1}{2} \psi_{t t}+|\psi|^{2} \psi+\frac{2}{3}|\psi|^{2} \phi+\frac{1}{3} \phi^{2} \psi^{*}=0,
\end{aligned}
$$

where $\phi$ and $\psi$ are the normalized orthogonal components of the optical field defined at the fiber input by $(\phi, \psi)=(1, i) U / \sqrt{2}$, and $\gamma$ is the half-difference between the propagation constants. Polarizer III mixes the two components into a single component, $U=\phi \cos (\theta)+\psi \sin (\theta)$. The latter is injected into the EDF. Observing the pulse at a certain point of the cavity at each round trip, we can see the pulse's convergence to some stationary profile.

The part of the cavity consisting of the birefringent fiber and the polarizer acts as a fast saturable absorber with a transmission coefficient that depends on the instantaneous intensity of the optical field. The laser starts to generate pulses at $\gamma$ near 0.01 and $\theta$ from $66^{\circ}$ to $142^{\circ}$. This range changes at higher values of $\gamma$. Once these parameters are chosen, we need to find the range of values where the two-pulse generation occurs. The double-pulse generation requires more intracavity energy.

One of the main consequences of periodic changes of the soliton shape is the radiation of small amplitude waves at fixed frequencies located symmetrically at each side of the soliton spectrum in the form of sidebands. Spectral sidebands related to periodic perturbation of a soliton were described in the original paper by Kelly. ${ }^{12}$ More detailed theory based on perturbation theory of solitons ${ }^{13}$ was presented later in Ref. 14. This effect is similar to Cherenkov radiation by solitons. ${ }^{15}$ The radiation serves as a bath for solitons in the cavity and can relate the separation and phase differences between the solitons. Because of the losses, the radiation field is decaying exponentially out of the soliton. Hence the interaction between the solitons will also be the strongest when solitons are located close to each other. At the same time, the interaction between the solitons will have periodic components because of the specific wavelength of radiation fields. This periodicity in the case of fiber links was noted in Ref. 10. Clearly, it should exist in the case of multiple-pulse generation by lasers.

We solve Eqs. (1) and (2) taking as the initial condition an arbitrary localized input. Fixing the cavity parameters and choosing the proper angle of the polarizer $\theta$, we observe a solution that converges to a stationary state consisting of one or several solitons. When the two-pulse solution appears, its relative phase difference $(\alpha)$ and separation $(\rho)$ converge to fixed values.

To find more two-soliton stationary states with different values of the phase difference and separation between solitons we start simulations with two single solitons of Eqs. (1) and (2) at arbitrary relative phase and distance. The single-soliton solutions are obtained for the same cavity parameters but with half the gain required for observation of two-soliton solutions. We then let them evolve until a stationary solution is reached. These simulations are repeated with many initial values of $\left(\rho_{0}, \alpha_{0}\right)$. Each stationary state has a basin of attraction to which the two-pulse solutions converge. The final values of $\rho$ and $\alpha$ for 15 stationary states are shown by the filled circles in Fig. 2. The phase difference for each of these states is close but not equal to $\pi / 2$. It oscillates as the number $N$ increases, approaching the limiting value of $\pi / 2$.

Figure 3 shows the spectra of the two-soliton solutions produced in the numerical simulations just after the polarizer. The solid curve corresponds to a

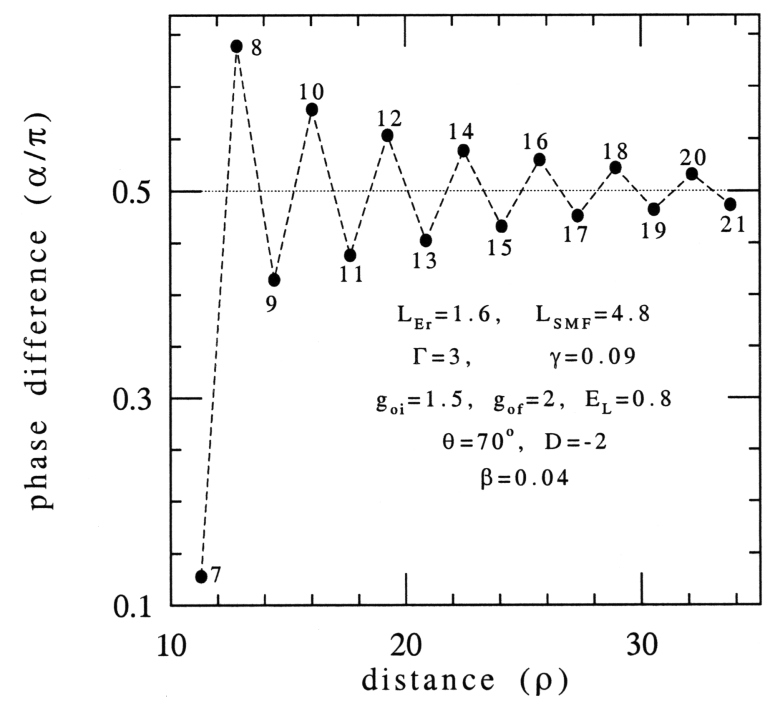

Fig. 2. Phase difference versus soliton separation for a discrete set of stable pairs obtained for the same cavity parameters.

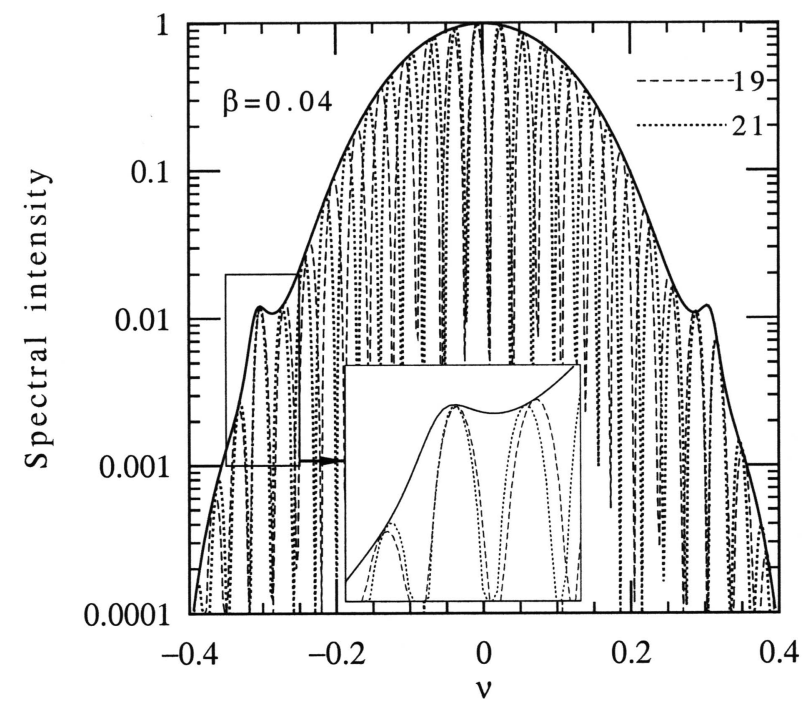

Fig. 3. Numerical spectra of the two-soliton solutions denoted 19 and 21 in Fig. 2. The solid curve corresponds to the spectrum of a single pulse. 


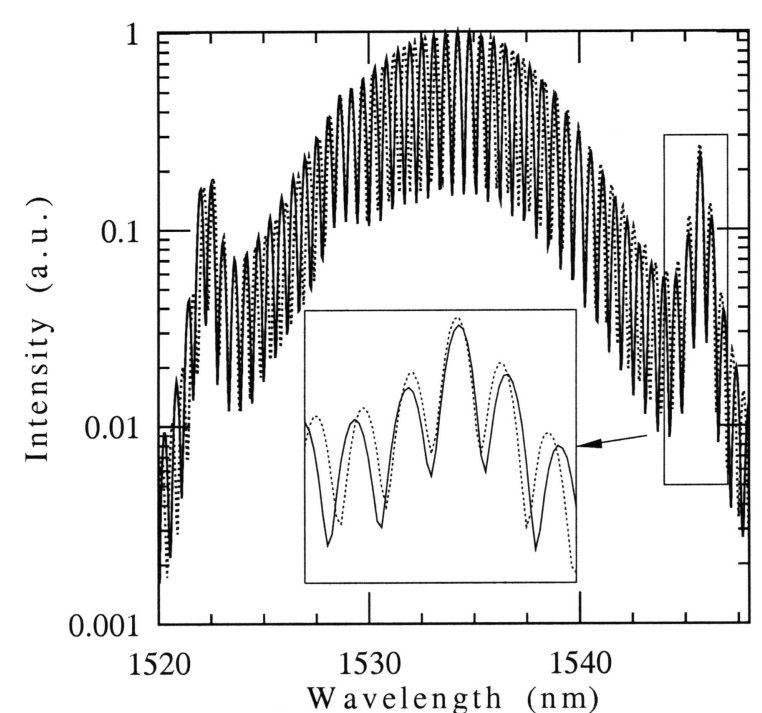

Fig. 4. Experimental spectra of the two-soliton solution.

single-soliton solution. The two strongest Kelly sidebands on each side of the main maximum are clearly seen. The spectra for two-soliton solutions are the result of interference between the two single-soliton spectra and therefore show the fringe pattern with a frequency that is the inverse of the pulse separation. The envelope of these spectra always coincides with the single-pulse spectrum. The two spectra for soliton pairs correspond to the solutions numbered as $N=19$ and $N=21$ in Fig. 2 . One can see clearly that one of the fringes coincides exactly with the left-hand Kelly sideband (solutions numbered with even $N$ possess a fringe coinciding with the right-hand Kelly sideband). Each successive stable solution has a fringe centered in one of the Kelly sidebands. The accuracy of this centering is higher for larger $N$. This observation proves that radiation related to the Kelly sidebands plays one of the major roles in the soliton coupling. It is remarkable that the phase difference between the solitons is fixed to $\pi / 2$, which makes the solution asymmetric so that only one side of the spectrum is attached to the sideband. Taking into account the asymmetry above, we note that the discrete time separation $\rho_{N}$ between the centers of the two solitons (filled circles in Fig. 2) can be easily calculated as $\rho_{N}=(2 N-1) /\left(4 \delta \nu_{1}\right)$, where $\delta \nu_{1}$ is the frequency displacement of the lowest-order Kelly sideband.

The relation between the Kelly sidebands and the quantized separations between solitons is further confirmed experimentally. The experimental setup that we are using is the same as in Ref. 2. It is clear from the analysis above that the spectral observations are sufficient for conclusive judgment. Figure 4 shows the two spectra for two different soliton-pair generation processes obtained for the same cavity parameters. First, one can notice that Kelly sidebands are much stronger in the experiment than in our numerical simulations. This is due to the unavoidable differences between the model and the real experimental setup. The stronger radiation allows further separation between the solitons that are still coupled to each other. The number of fringes between the two sidebands differs also from those in the numerical simulations. Despite these differences, the spectral peaks on the right-hand sides of the two spectra exactly coincide with the peak of the Kelly sideband. This fact confirms our numerical findings that the two-pulse separation takes quantized values that are related to the Kelly sidebands. Another fact that agrees with our numerical results is that the spectra are asymmetric. This means that the phase difference between the solitons is $\sim \pi / 2$ rather than 0 or $\pi$.

N. Akhmediev acknowledges financial support from the Secretaría de Estado de Educación y Universidades, Spain, Reference No. SAB2000-0197, and the Australian Research Council. The work of J. M. Soto-Crespo (e-mail, iodsc09@io.cfmac.csic.es) was supported by the Spanish Ministerio de Ciencia y Tecnología under contract BFM2000-0806.

\section{References}

1. A. B. Grudinin, D. J. Richardson, and D. N. Payne, Electron. Lett. 28, 67 (1992).

2. Ph. Grelu, F. Belhache, F. Gutty, and J. M. SotoCrespo, Opt. Lett. 27, 966 (2002).

3. M. Lai, J. Nicholson, and W. Rudolf, Opt. Commun. 142, 45 (1997).

4. M. J. Lederer, B. Luther-Davies, H. H. Tan, C. Jagadish, N. N. Akhmediev, and J. M. Soto-Crespo, J. Opt. Soc. Am. B 16, 895 (1999).

5. H. Kitano and S. Kinoshita, Opt. Commun. 157, 128 (1998).

6. N. N. Akhmediev, A. Ankiewicz, and J. M. Soto-Crespo, J. Opt. Soc. Am. B 15, 515 (1998).

7. E. E. Dianov, A. V. Luchnikov, A. Pilipetskii, and A. M. Prokhovov, J. Appl. Phys. B 54, 175 (1992).

8. A. N. Pilipestskii and C. R. Menyuk, Opt. Lett. 21, 119 (1996).

9. J. N. Kutz, B. C. Kollings, K. Bergman, and W. H. Knox, IEEE J. Quantum Electron. 34, 1749 (1998).

10. L. Socci and M. Romagnoli, J. Opt. Soc. Am. B 16, 12 (1999).

11. E. A. Kuznetsov, A. V. Mikhailov, and I. A. Shimokhin, Physica D 87, 201 (1995).

12. S. M. J. Kelly, Electron. Lett. 28, 806 (1992).

13. J. P. Gordon, J. Opt. Soc. Am. B 9, 91 (1992).

14. J. N. Elgin and S. M. J. Kelly, Opt. Lett. 18, 787 (1993).

15. N. N. Akhmediev, A. V. Buryak, and M. Karlsson, Opt. Commun. 110, 540 (1994). 